CORRECTION

https://doi.org/10.1038/s41586-019-1458-y

\title{
Publisher Correction: Group 3 innate lymphoid cells mediate early protective immunity against tuberculosis
}

Amanda Ardain, Racquel Domingo-Gonzalez, Shibali Das, Samuel W. Kazer, Nicole C. Howard, Alveera Singh, Mushtaq Ahmed, Shepherd Nhamoyebonde, Javier Rangel-Moreno, Paul Ogongo, Lan Lu, Duran Ramsuran, Maria de la Luz Garcia-Hernandez, Tyler K. Ulland, Matthew Darby, Eugene Park, Farina Karim, Laura Melocchi, Rajhmun Madansein, Kaylesh Jay Dullabh, Micah Dunlap, Nancy Marin-Agudelo, Takashi Ebihara, Thumbi Ndung'u, Deepak Kaushal, Alexander S. Pym, Jay K. Kolls, Adrie Steyn, Joaquín Zúñiga, William Horsnell, Wayne M. Yokoyama, Alex K. Shalek, Henrik N. Kløverpris, Marco Colonna, Alasdair Leslie \& Shabaana A. Khader

Correction to: Nature https://doi.org/10.1038/s41586-019-1276-2, published online 05 June 2019.

In Fig. 2a of this Letter, owing to an error during the production process, the $y$-axis label of the right-most graph was incorrectly shown as 'No. of ILC3s per lung $\left(\times 10^{3}\right)$ ' instead of 'No. of ILC3s per lung $\left(\times 10^{2}\right)$ '. In addition, author Marco Colonna was inadvertently associated with affiliation 7 (Division of Allergy, Immmunology and Rheumatology, Department of Medicine, University of Rochester Medical Center, Rochester, NY, USA) instead of affiliation 9 (Division of Immunobiology, Department of Pathology and Immunology, Washington University School of Medicine, St Louis, MO, USA). These errors have been corrected online. 